\title{
Fluid Dynamic Analysis on the Adaptive Slot Method of Hypersonic Inlets
}

\author{
Chengxiang Zhu* and Yancheng You \\ School of Aerospace Engineering, Xiamen University, China \\ ${ }^{*}$ Corresponding author
}

\begin{abstract}
To enhance the starting ability was one of the most serious issues in air propulsion system. In the present work, an adaptive slot control method was proposed to expand the working range of hypersonic inlets to a lower Mach number with weak losses, which was beneficial for engineering applications. Simulation results indicated that the starting Mach number can be decreased from 3.2 to 3.0 with five parallel slots at a width of $4 \mathrm{~mm}$. The flow inside the slot channel was self-driven due to the pressure gradient across of the separation shock. In case that the inlet was restart, the slot channel flow would be strongly suppressed.
\end{abstract}

Keywords-adaptive slot; hypersonic inlet; starting ability; restart; separation

\section{INTRODUCTION}

The starting characteristics of hypersonic inlets directly determine the operational range of the propulsion system, and highly affect the flight safety. The capability of easy selfrestarting during accidental unstart is hence a worldwide research interest. For most hypersonic inlet unstart, the shock/boundary-layer interaction leads to the generation of separation bubbles along the wedge surface near the cowl lip, which narrows the actual flow path of the inlet ${ }^{[1]}$. And as a consequence, the mass flow ratio and the total pressure recovery of the inlet decrease significantly, resulting in a performance deterioration of the propulsion system. Xie had developed a flow-based method to predict the self-starting limit of two-dimensional hypersonic inlets, in which the migration of the separation bubble was considered ${ }^{[2]}$.

To minimize the influence of inlet unstart, many selfrestarting approaches have been suggested. Variable geometry was one of the efficient ways to expand the operation range of hypersonic inlets. By rotating the cowl lip at off-design points, Jin successfully reduced the starting Mach number to 3.5 for a hypersonic inlet designed at Mach $6^{[3]}$. Falempin investigated a combined variable inlet/combustor cowl which could adapt the contraction ratio to the flight conditions from Mach 2 to $8^{[4]}$. Zhang proposed a moveable slot-plate control method to solve hypersonic inlet unstart ${ }^{[5]}$. For fixed geometries, Schulte implemented a boundary layer bleed system to decrease the interaction between shock wave and boundary layer ${ }^{[6,7]}$. The bleeding air in the boundary layer forced the airflow to deflect outwards, generating a series of compression waves which eventually affected the incident shock and promoted the inlet starting. Similar techniques can also be found in ${ }^{[8-13]}$. Yuan presented a reverse suction method to analyze its influence on the self-starting ability ${ }^{[14,15]}$. Nevertheless, the bleed or suction system utilized by them complicated the whole structure of the inlet and caused mass loss. Li proposed a pulse-starting method to promote the inlet starting capability, in which a fairing was used before the engine was brought to a certain speed ${ }^{[16]}$. The drawback however lies in the weight increment of the fairing and the need of an additional propulsion system. In order to control inlet flow separation, the Magneto-hydrodynamic technique (MHD) was adopted in some applications. By introducing plasma ahead of the separation bubble, the flow velocity inside the boundary layer can be controlled by MHD [17-19]. The momentum of the boundary layer flow can hence be adjusted to change the bubble size and location, which further affected the inlet starting feature. The control efficiency of MHD actually depends highly on flight speed. To achieve similar control efficiency, more electric energy was required at higher speed.

The above mentioned methods all performed nicely in inlet restarting. However, either complex geometry structure, or dramatic weight increment, or obvious energy loss limited their practical applications. How to achieve higher restart capability with lower effort is still an open question. Wang recently proposed a fluidic control method for improving the selfstarting ability of hypersonic inlets ${ }^{[20]}$. This was an advisable approach, but the entrance and exit of the backflow duct they applied was vertical to the incoming flow, which would lead to obvious inlet performance reduction. In the present work, an improved method with series of adaptive slots was proposed to improve the inlet starting characteristics. This method is easy to actualize and does not cause weight increment since the pressure difference inside the inlet will generate circulation flow automatically.

\section{NUMERICAL METHOD AND SETUPS}

\section{A. Numerical Tool}

The numerical tool utilized in the present work was the commercial software Ansys Fluent V14.0. A series of twodimensional numerical simulations were carried out based on the compressible Reynolds averaged Navier-Stokes equations as in the following form, in which $\rho, p, t, e$ indicate density, pressure, time, and internal energy, respectively, $u, S, \mathbf{q}$ are velocity, stress tensor, and heat flux, respectively.

$$
\frac{\partial \rho}{\partial t}+\nabla \cdot(\rho \mathbf{u})=0
$$




$$
\frac{\partial(\rho \mathbf{u})}{\partial t}+\nabla \cdot(\rho \mathbf{u}) \otimes \mathbf{u}=-\nabla p+\nabla \cdot \mathbf{S}
$$

$$
\frac{\partial}{\partial t}\left(\frac{1}{2} \rho \mathbf{u}^{2}+\rho e\right)+\nabla \cdot\left[\mathbf{u}\left(\frac{1}{2} \rho \mathbf{u}^{2}+\rho e\right)\right]=-\nabla \cdot \dot{\mathbf{q}}-\nabla \cdot(p \mathbf{u})+\nabla \cdot(\mathbf{u} \cdot \mathbf{S})
$$

To solve the momentum equations, the two-equation SST (shear-stress transport) $k$ - $\omega$ turbulence model was used for the turbulent viscosity term. The flux term was solved with RoeFDS (Roe flux-difference splitting) scheme. The $1^{\text {st }}$ order upwind scheme was adopted to discretize the equations spatially in order to get a stable initial flow field firstly, and the $2^{\text {nd }}$ order upwind scheme was used afterwards to accurate the flow.

\section{B. Hypersonic Inlet Model}

For the present study, a base hypersonic inlet was designed at Mach 5 and altitude of $23 \mathrm{~km}$, the external compression part of which was composed of a wedge surface and an isentropic compression surface. The height of the inlet was $250 \mathrm{~mm}$, the throat height was $71.35 \mathrm{~mm}$, and the internal compression ratio was 1.32. A series of parallel adaptive slots were arranged on the isentropic compression surface with an injection angle of 45 degree. The slot depth was designed as $15 \mathrm{~mm}$, and the distance between each two slots was $50 \mathrm{~mm}$, as shown in Fig. 1. The slot width was set as $4 \mathrm{~mm}$. Pressure-far-field was set for both inflow and surrounding faces, whereas pressure-outlet was set as the exit boundary condition for both slots and the entire inlet model. A structured grid with a total number of 0.4 million was used for the current two-dimensional geometry. Grid refinement was applied near the surface wall $\left(y^{+}=2\right)$, especially near the slots to capture the flow feature more accurately.

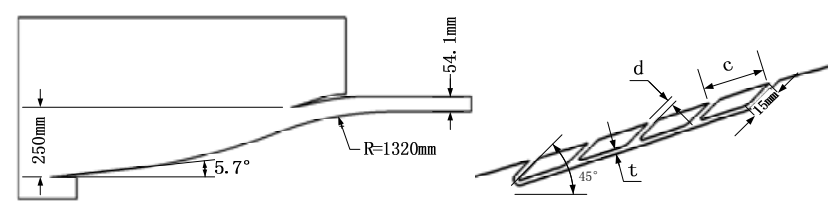

FIGURE I. HYPERSONIC INLET MODEL

\section{STARTING CAPABILITY}

\section{A. Inlet without Slots}

To determine the starting capability of the base hypersonic inlet, a series of simulations were conducted at Mach number 2.7, 2.8, 2.9, 3.0, 3.1 and 3.2, as shown in Fig. 2. Obviously, the inlet remained unstart below Mach 3.2. Within the unstart regime, a large separation bubble was generated near the cowl lip on the lower surface, which hence resulted in a strong separation shock there. Meanwhile, a narrowed gaseous throat can be observed near the cowl lip, which decreased the flow speed to only sonic level. In addition, a weak shock train inside the inlet was generated, forcing the upper boundary layer to separate as well. For any Mach number not larger than 3.1, the unstart phenomena of the inlet were quite similar. Up to Ma =3.2, however, a starting flow field with clear shock wave reflections was obtained, where the separation bubble was swallowed by the inlet. Therefore, the starting Mach number of the base hypersonic inlet was determined as 3.2.
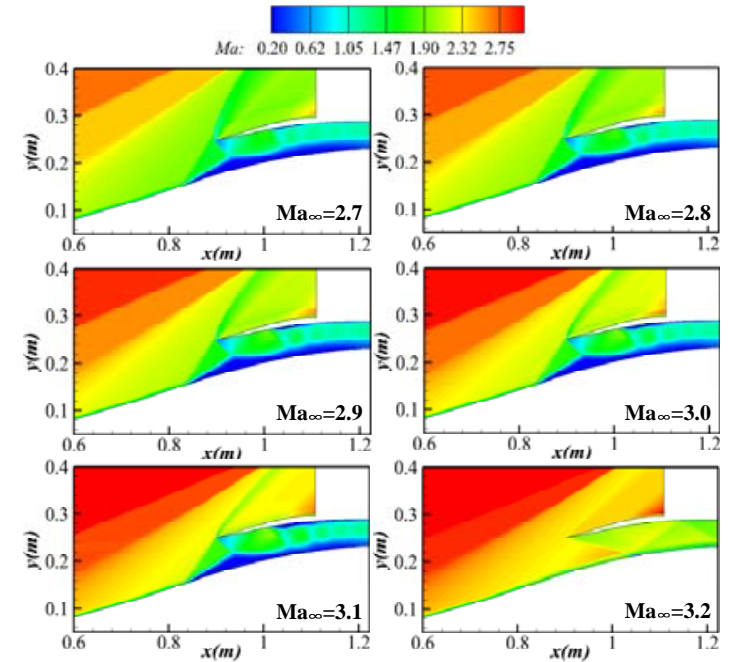

FIGURE II. PERFORMANCE OF THE INLET WITHOUT SLOTS

\section{B. Inlet with Slots}

The five adaptive slots were arranged on the external compression surface to improve the starting characteristics of the base hypersonic inlet, as shown in Fig. 3. In order to achieve a better control efficiency, the middle slot was arranged at the location of the separation shock. The two downstream slots were built within the separation bubble, whereas the two upstream slots were forehead of the separation bubble. At $\mathrm{Ma}_{\infty}=2.7$, the inlet was unstart, with a large separation bubble developed on the lower surface. A similar shock train as that in Fig. 5 can be detected within the internal part of the inlet. Outside the cowl lip, however, a series of compression waves due to the existence of slots were generated, mixing with the separation shock. And the flow speed remained all subsonic inside the slot channels. These flow phenomena were similar at $\mathrm{Ma}_{\infty}=2.8$. Nevertheless, with increasing Mach number to 2.9, the separation bubble moved backwards slightly, covering only two downstream slots. This separation bubble was further swallowed by the inlet at higher Mach number 3.0, resulting in a restarting flow field where clear shock reflections were already formed within the inlet.

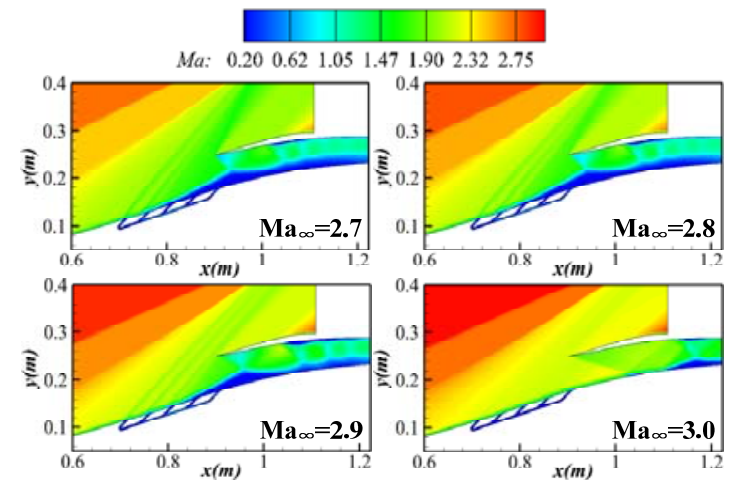

FIGURE III. PERFORMANCE OF THE INLET WITH SLOTS 
With the application of adaptive slots, a significant improvement of inlet starting capability was obtained, in which the starting Mach number decreased from 3.2 to 3.0. The flow inside the slot channels was self-driven due to pressure gradient. High pressure gas in the separation bubble with low kinetic energy was sucked into the two downstream slots, and was then injected from the upstream slots back into the flow with a higher velocity. This actually had two positive effects on the starting capability. Firstly, the separation region shrunk due to the suction of downstream slots. The actual effective throat area was hence enlarged, promoting the inlet restarting. Secondly, the flow injection from the upstream slots led to the formation of compression waves, which tended to discharge more mass flow out of the inlet and promoted the inlet restarting as well. The flow structure of the inlet with adaptive slots was provided in Fig. 4. It should be emphasized that, as a self-adaptive method, the suction and injection flow rate was self-controlled. With decreasing size of the separation bubble during restarting process, the strength of the separation shock weakened accordingly. Thus, the pressure gradient inside the slot system reduced as a result, which also meant a lower flow rate.

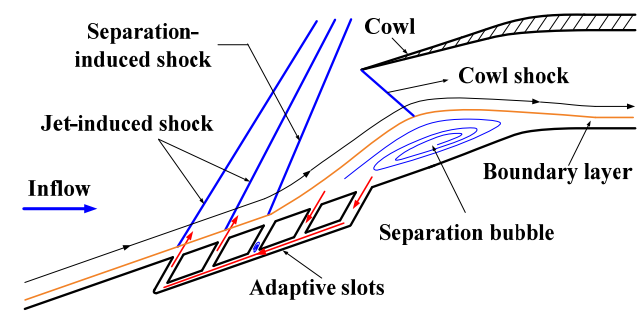

FIGURE IV. INLET FLOW CHARACTERISTICS

The fundamental physical mechanism of adaptive slots was the pressure gradient within different slots which promoted the channel flow. The arrangement of the slots covered across the separation shock ahead of the separation bubble, which generated higher pressure within the bubble zone in downstream slots, whereas the pressure in upstream slots was relatively lower. It should be emphasized that the pressure difference was self-adapted depending on the location and strength of the separation shock. For inlet restarting, the channel flow would be significantly suppressed due to absence of the separation bubble.

\section{Unsteady Feature}

An unsteady simulation was conducted in this section to analyze the physical mechanism of the adaptive slots. Five slots with width $d=4 \mathrm{~mm}$ were selected for the current analysis at Ma ${ }_{\infty}=3.0$. The flow contours of the inlet at three time instants were shown in Fig. 5, associated with zoomed in views. At $t_{l}$, the five slots were all covered by low energy region, where the separation shock was generated ahead of the slots. The separation bubble was nearly half of the throat height at this time. Regarding to the streamlines, the two downstream slots sucked in some low energy gas, whereas injecting from the two upstream slots, forming a circulation flow. With increasing time to $t_{2}$, the separation bubble moved backwards, the separation shock of which was located near the third slot. The downstream three slots were still within the low energy region at the current time, whereas the upstream two slots were already exposed to high speed incoming flow. The size, especially the length of the separation bubble was significantly decreased. To $t_{3}$, the bubble size was even decreasing, leading to a throat area sufficient for all captured incoming flow. The inlet was thus restarted, where all five adaptive slots were forehead of the separation shock.

The unsteady process aforementioned actually indicated two important things. Firstly, the main positive effect of slots on inlet starting capability lied on the bubble size decreasing. Once an actual throat area sufficient for the captured incoming flow was obtained, the inlet would restart. Secondly, these slots were all self-adaptive according to the location of the separation shock. The pressure gradient before and after the separation shock led to automatic gaseous suction or injection. Therefore, no more accessary system was required, which is also one of the dominant advantages of the current proposed method.

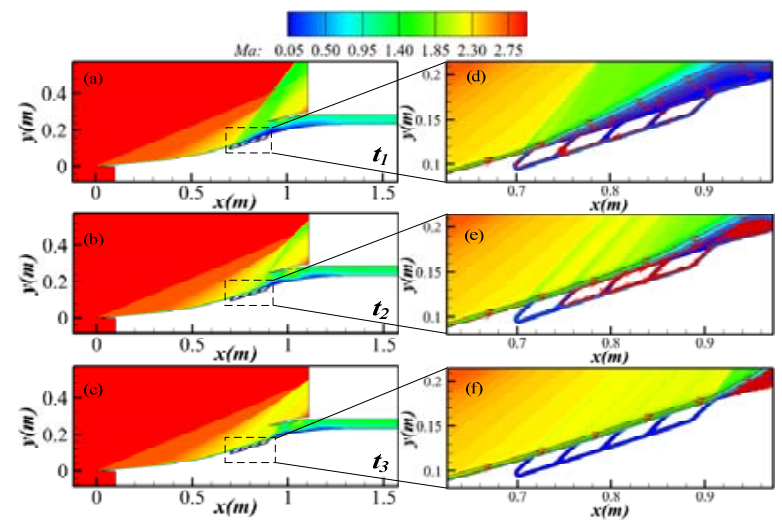

FIGURE V. INLET UNSTEADY FEATURE

\section{DISCUSSION}

Adaptive slots showed a nice efficiency on promoting the starting characteristics of hypersonic inlets. By carefully arranging the location of slots, a self-driven circulation flow could be generated within the channel once the inlet was unstarting. Furthermore, this circulation flow was self-adaptive at different Mach numbers. In case of inlet restarting, this flow behavior would be terminated due to weak driven pressure gradient.

From the above analysis, two dominant advantages of adaptive slots can be addressed. Firstly, no additional weight: This method requires no external structures or facilities to drive the flow. Secondly, no mass loss: Gases sucked into the downstream slots from the separation bubble with high pressure and low kinetic energy, were injected back into the flow from the upstream slots. One more important feature for this method is that the starting Mach number keeps nearly the same for the investigated three different slot widths, i.e. no significant influence of slot width on the starting capability. Furthermore, by reducing the slot number from five to three, the starting feature also remains similar. That is to say, the dependence of the inlet starting characteristics on either slot width or slot number is weak. Once the location of the separation bubble is identified, one single slot within the 
bubble and the other slot ahead of the bubble would be sufficient as well as efficient. This would be an interesting and also important finding for the simple adaptive slot method.

\section{ACKNOWLEDGMENT}

The authors would like to thank the National Natural Foundation of China (51606161), and the Fundamental Research Funds for the Central Universities (20720170055) for the financial support of this project.

\section{REFERENCES}

[1] Yan, H., Zhong, J., Han, J., Feng, Z., and Yu, Y., "Research on Boundary Layer Suction in the Throat of Supersonic Inlet," Journal of Propulsion Technology, Vol. 30, No. 2, 2009, pp. 175-181 (in Chinese).

[2] Xie, W., Ma, G., Guo, R., Chen, H., Wen, Y., and Guo, S., "Flow-based Prediction for Self-starting Limit of Two Dimensional Hypersonic Inlets,” Journal of Propulsion and Power, Vol. 32, No. 2, 2016, pp. 463471.

[3] Jin, Z., Zhang, K., Chen, W., and Liu, Y., "Design and Regulation of Two-dimensional Variable Geometry Hypersonic Inlets,” Acta Aeronautica et Astronautica Sinica, Vol. 34, No. 2, 2013, pp. 779-786 (in Chinese).

[4] Falempin, F., Wendling, E., Goldfeld, M., and Starov, A., "Experimental Investigation of Starting Process for a Variable Geometry Air Inlet Operating from Mach 2 to Mach 8,” AIAA Paper 2006-4513, 2006.

[5] Zhang, Q., Tan, H., Bu, H., and Rao, C., "Investigation of a Moveable Slot-plate Control Method for Hypersonic Inlet Unstart Caused by Downstream Mass-flow Choking,” AIAA Paper 2014-3847, 2014.

[6] Schulte, D., Henckels, A., and Wepler, U., "Reduction of Shock Induced Boundary Layer Separation in Hypersonic Inlets Using Bleed," Aerospace Science and Technology, Vol. 2, No.4, 1998, pp. 231-239.

[7] Schulte, D., Henckels, A., and Neubacher, R., "Manipulation of Shock/Boundary-Layer Interactions in Hypersonic Inlets," Journal of Propulsion and Power, Vol. 17, No.3, 2001, pp. 585-590.

[8] Haws, R., Noall, J., and Daines, R., "Computational Investigation of a Method to Compress Air Fluidically in Supersonic Inlets," Journal of Spacecraft and Rockets, Vol. 38, No. 1, 2001, pp. 51-59.

[9] You, J., Xia, Z., Fang, C., Hu, J., and Liu, B., "Influence of Single Square Pulse Injection on Restart Characteristics for Mixed Compression Inlet,” Journal of Aerospace Power, Vol. 26, No. 10, 2011, pp. 2181-2187 (in Chinese).

[10] Tan, H.J., Li, C.H., and Zhang, Y., "Investigation of a Fluidic Shock Control Method for Hypersonic Inlets," Journal of Propulsion and Power, Vol. 26, No. 5, 2010, pp. 1072-1083.

[11] Haberle, J., and Gulhan, A., "Investigation of the Performance of a Scramjet Inlet at Mach 6 with Boundary Layer Bleed," AIAA Paper 2006-8139, 2006.

[12] Soltani, M., Daliri, A., Younsi, J., and Farahani, M., "Effects of Bleed Position on the Stability of a Supersonic Inlet," Journal of Propulsion and Power, Vol. 32, No. 5, 2016, pp. 1153-1166.

[13] White, M., Lee, R., Thompson, M., and Carpenter, A., "Tangential Mass Addition for Shock/Boundary Layer Control in Scramjet Inlets,” Journal of Propulsion and Power, Vol. 7, No. 6, 1991, pp. 1023-1029.

[14] Yuan, H., and Liang, D., "Effect of Suction on Starting of Hypersonic Inlet," Journal of Propulsion Technology, Vol. 27, No. 6, 2006, pp. 525528 (in Chinese).

[15] Wang, W., Yuan, H., Huang, G., and Liang, D., "Impact of Suction Position on Starting of Hypersonic Inlets," Journal of Aerospace Power, Vol. 24, No. 4, 2009, pp. 918-924 (in Chinese).

[16] Li, Y., He, G., and Liu, P., "Investigation on One Aid Hypersonic Inlet Starting Method,” Journal of Solid Rocket Technology, Vol. 30, No.5, 2007, pp. 392-395 (in Chinese)

[17] Fang, C., Xia, Z., Hu, J., and You, J., “Advances in Inlet Flow Control Technology,” Missiles and Space Vehicles, No. 2, 2014, pp. 34-38 (in Chinese).
[18] Fomin, V., Tretyakov, P., and Taran, J., "Flow Control Using Various Plasma and Aerodynamic Approaches," Aerospace Science and Technology, Vol. 8, No. 5, 2004, pp. 411-421.

[19] Caraballo, E., Web, N., and Little, J., "Supersonic Inlet Flow Control Using Plasma Actuators,” AIAA Paper 2009-924, 2009.

[20] Wang, J., Xie, L., Zhao, H., Teng, Y., and Ma, G., "Fluid Control Method for Improving the Self-starting Ability of Hypersonic Inlets," Journal of Propulsion and Power, Vol. 32, No. 1, 2016, pp. 153-160. 